\title{
Tuberculosis of Proximal Humerus with Biceps Tendon Involvement - A Rare Case Report
}

\author{
Dr. Neetin P Mahajan ${ }^{1}$, Dr. Tushar C Patil ${ }^{2 *}$, Dr. Kartik Pande ${ }^{2}$, Dr. Kunal Chaudhari ${ }^{2}$
}

${ }^{1}$ Professor and Unit Head, Department of Orthopaedics, Grant Government Medical College, Mumbai, Maharashtra, India

${ }^{2}$ Junior Resident, Department of Orthopaedics, Grant Government Medical College, Mumbai, Maharashtra, India

DOI: $10.36347 /$ simcr.2022.v10i01.004

| Received: 13.12.2021 | Accepted: 05.01.2022 | Published: 14.01.2022

*Corresponding author: Dr. Tushar C Patil

Junior Resident, Department of Orthopaedics, Grant Government Medical College, Mumbai, Maharashtra, India

Abstract

Case Report

Introduction: Extra pulmonary TB is caused by dissemination of primary infection through blood and lymph to neighbouring regions like pleura, lymph nodes, bones and joints, where they remain dormant. Reactivation of the disease is caused by disorders of immune system. Skeletal TB consists of $2 \%$ of all TB cases and $10-35 \%$ of extrapulmonary TB cases. Skeletal TB in humerus are a rare occurrence accounting upto 1-2\% of total skeletal TB cases. Here we present a case of proximal humerus tuberculosis which was misdiagnosed and treated as rotator cuff injury by local practitioners and adviced physiotherapy. Case report: The patient is a 16 years old female with humeral head osteomyelitis at bone tendon interface with biceps tendon involvement. We managed the case with MRI followed by CT guided biopsy and anti tubercular regimen was started based on histopathology reports. Patient had resolution of infection with good range of shoulder mobility and satisfactory function. Conclusion: The diagnosis of proximal humerus TB is clinically challenging due to confluence of nonspecific symptoms and X ray findings which misguides clinicians to misdiagnose it as rotator cuff pathology, frozen shoulder, pyogenic osteomyelitis or tumours. This leads to delay in treatment. Probability of skeletal TB should always be kept in mind in such cases, especially in endemic countries which will help in timely diagnosis and treatment.

Keywords: Extra pulmonary, skeletal, tuberculosis (TB), proximal humerus, humeral head, biceps tendon involvement.

Copyright $\odot 2022$ The Author(s): This is an open-access article distributed under the terms of the Creative Commons Attribution 4.0 International License (CC BY-NC 4.0) which permits unrestricted use, distribution, and reproduction in any medium for non-commercial use provided the original author and source are credited.

\section{INTRODUCTION}

Extra pulmonary TB is caused by dissemination of primary infection through blood and lymph to neighbouring regions like pleura, lymph nodes, bones and joints, where they remain dormant. Reactivation of the disease is caused by disorders of immune system [1].

Skeletal TB consists of $2 \%$ of all TB cases and $10-35 \%$ of extrapulmonary TB cases [2].

Osteoarticular TB is more common in endemic countries with high prevalence amongst children and young adults. Acute fulminating type of skeletal TB is common in children where as dry type of skeletal TB is common in adults. It most commonly affects spine $(50 \%)$, followed by hip (15\%) and knee joints $(12 \%)$. Skeletal TB in humerus are a rare occurrence accounting upto $1-2 \%[1,3,4]$.
The global burden of TB is approximately 30 million, of which one third cases are from India [3]

Proximal humerus TB being rare, it can easily be misdiagnosed and neglected due to non specific clinical findings which resembles frozen shoulder. Radiologically it resembles pyogenic osteomyelitis [3, 5].

Chronic mono articular shoulder pain can include differential diagnosis as shoulder impingement syndrome, Brodie's abscess, chronic pyogenic osteomyelitis and neoplasms [6].

\section{Case Report}

A 16 years old female came with left shoulder pain since 1 year which was insidious in onset and gradually progressive. She had previously taken treatment from outside where she was managed with painkillers and physiotherapy. Since there was no relief 
Neetin P Mahajan et al., Sch J Med Case Rep, Jan, 2022; 10(1): 17-20

patient was came to us for further management. On shoulder examination, patient had painful restrictions of biceps movement. There was no history of trauma or any constitutional TB symptoms. Plain radiographs showed focal osteopenia and lytic area in proximal humerus as seen in Fig 1. We adviced for MRI which showed periarticular edema and erosions with biceps tendon involvement as seen in Fig 2. CT guided biopsy from left proximal humerus was done which came positive for Mycobacterium tuberculosis on histopathological examination as seen in Fig 3 and AKT regimen was started according to fixed dose combination.

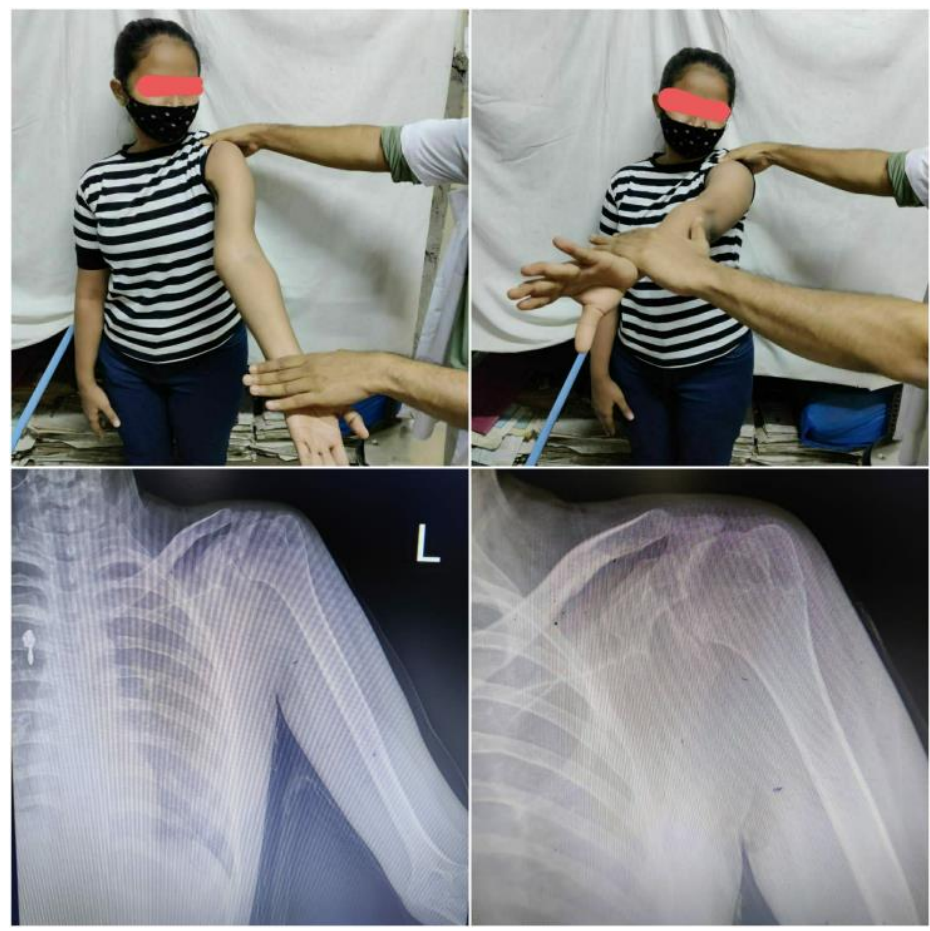

Figure 1: At presentation, clinical pictures showing weakness of biceps tendon and $\mathrm{X}$ rays showing focal osteopenia and lytic lesion in proximal humerus

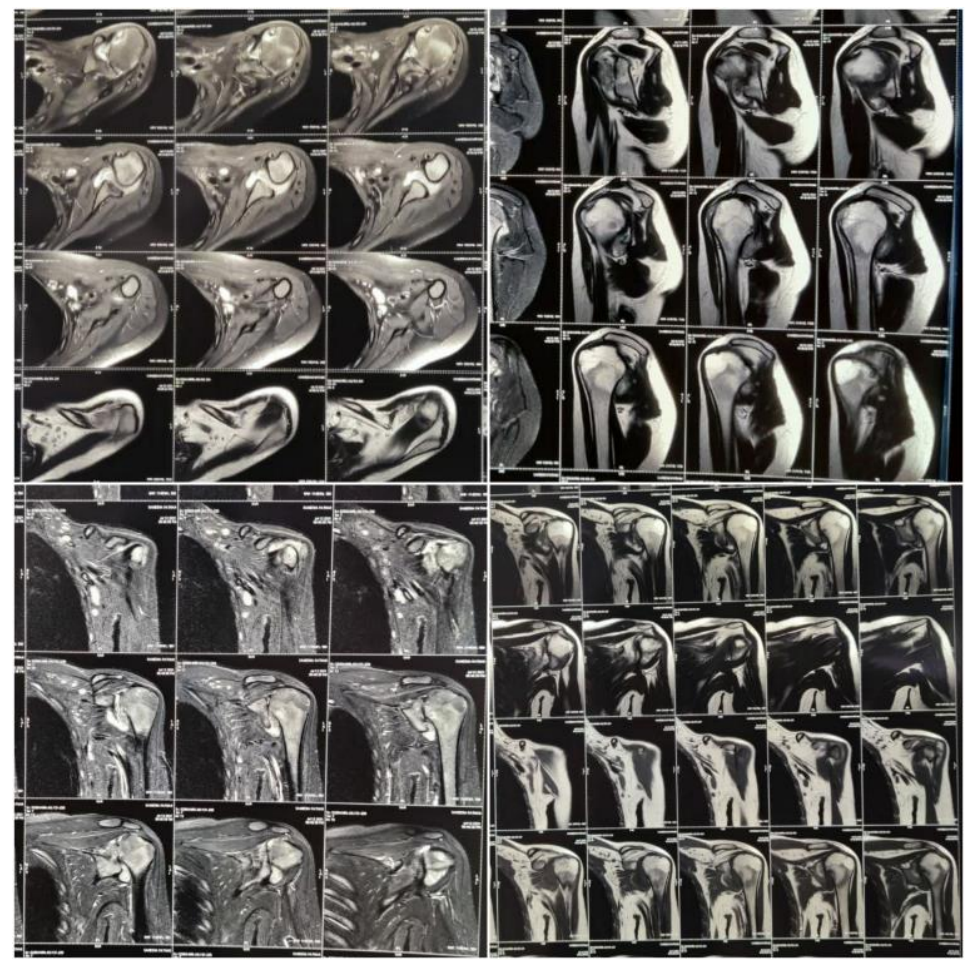

Figure 2: MRI left shoulder showing perisynovial edema, reduced joint space, articular erosions and biceps tendon involvement 
Neetin P Mahajan et al., Sch J Med Case Rep, Jan, 2022; 10(1): 17-20

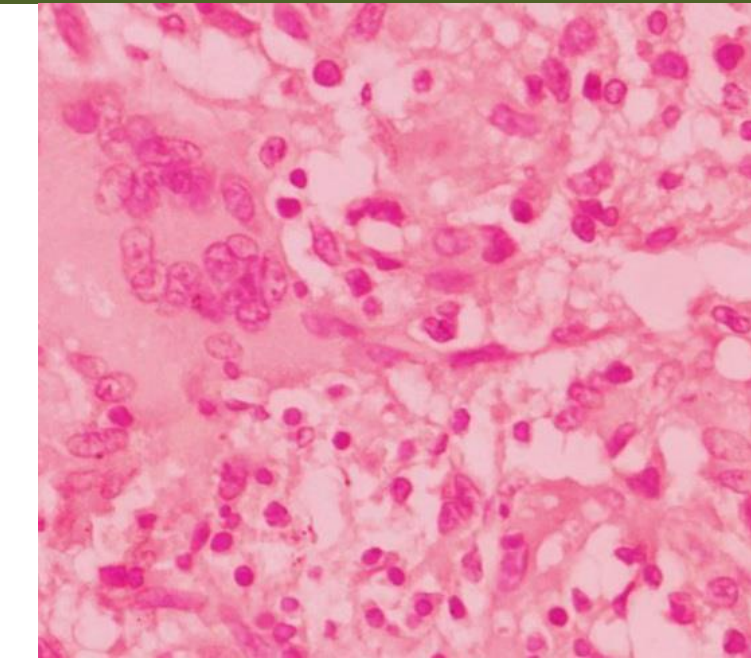

Figure 3: Biopsy sample showed Langerhan giant cells and caseous necrosis on histopathological examination

\section{DiscuSSION}

Recent rise in patients of extrapulmonary TB has been attributed to increase in pulmonary TB cases in HIV patients, as TB is 3 times more common in HIV positive patients. Along with emergence of resistant strains and emigration of workers from endemic countries has increased prevalence of tuberculosis even in developed western countries $[1,7]$.

Initial tubercular lesion occurs in the area of high vascularity and venous stasis causing colonization of aerobic tubercular bacteria followed by formation of granuloma. This is then accompanied by focal necrosis and destruction of surrounding bone and other tissues by osteoclasts and inflammatory cytokines. This then leads to forming gravity abscesses [1].

The course of the disease is slow, taking months to years for significant symptoms to manifest. It starts by diffuse pain in the affected joint which is radiated by surrounding nerves. This is followed by increase in swelling with increase local warmth and redness. Joint mobility is decreased. Night pain is characteristic of osteoarticular TB, which occurs when inflammed surfaces of joint rub against each other when surrounding muscles relax during sleep. This leads to disuse atrophy of surrounding muscles. Abscess leads to formation of sinuses. Sometimes only a painless cold abscess may be the presenting symptom [1].

On plain radiographs, skeletal TB shows features of osteopenia, joint space narrowing, subchondral erosions or focal lysis. Laboratory investigations may show increased leukocytes, erythrocyte sedimentation rate (ESR) and test for C reactive proteins (CRP). Evidence of granulomatous inflammation, caseous necrosis and Langerhan giant cells on histopathological testing remains the gold standard for diagnosing skeletal TB [2].
Maheshwar et al., have stressed on importance of fine needle aspiration cytology in diagnosis of osteoarticular TB mimicking tumours [4]

Radiological imaging like MRI, being simple and non invasive, is very important for early diagnosis of skeletal TB and its differentiation from pyogenic osteomyelitis or tumours [8].

We haven't found any literature specifying tuberculosis at bone tendon interface of humeral head with biceps tendon rupture.

Tuberculosis of tendon bone interface is very rare in literature. This region is under constant traction forces and microavulsions. There is slow healing response and requires longer time for remodeling to restore mechanical properties [7].

Drainage of caseation necrosis followed by anti tubercular medications is the line of management [8].

World Health Organization (WHO) recommends 4 drugs regimen for 2 months followed by 2 drug regimen for 7-10 months, for management of tuberculosis [6].

\section{CONCLUSION}

The diagnosis of proximal humerus TB is clinically challenging due to confluence of nonspecific symptoms and $\mathrm{X}$ ray findings which misguides clinicians to misdiagnose it as rotator cuff pathology, frozen shoulder, pyogenic osteomyelitis or tumours. This leads to delay in treatment. Probability of skeletal TB should always be kept in mind in such cases, especially in endemic countries which will help in timely diagnosis and treatment.

\section{Declaration of Patient Consent}

The authors certify that they have obtained all appropriate patient consent forms. In the form the patient(s) has/have given his/her/their consent for his/her/their images and other clinical information to be reported in the journal. The patients understand that their names and initials will not be published and due efforts will be made to conceal their identity, but anonymity cannot be guaranteed.

\section{Financial support and sponsorship: Nil.}

Conflicts of Interest: There are no conflicts of interest.

\section{REFERENCES}

1. Michałowska-Mitczuk, D., \& Błasińska-Przerwa, K. (2011). Tuberculosis of shoulder bone. Advances in Respiratory Medicine, 79(6), 437-441. 
Neetin P Mahajan et al., Sch J Med Case Rep, Jan, 2022; 10(1): 17-20

2. Hosalkar, H. S., Agrawal, N., Reddy, S., Sehgal, K., Fox, E. J., \& Hill, R. A. (2009). Skeletal tuberculosis in children in the Western world: 18 new cases with a review of the literature. Journal of children's orthopaedics, 3(4), 319-324.

3. Sahu, S., Pattnaik, S., Mohanty, I., Narasimham, M. V., \& Panda, P. (2013). Chronic osteomyelitis of humerus presenting as scrofuloderma. Indian journal of dermatology, 58(5), 408.

4. Mangwani, J., Gupta, A. K., Yadav, C. S., \& Rao, K. S. (2001). Unusual presentation of shoulder joint tuberculosis: a case report. Journal of Orthopaedic surgery, 9(1), 57-60.

5. Aggarwal, R., Roy, K., Nemade, P., \& Purohit, S. (2016). XDR-TB of the shoulder presenting as a

lytic lesion in the proximal humerus. The

International Journal of Tuberculosis and Lung Disease, 20(2), 283-284.

6. Longo, U. G., Marinozzi, A., Cazzato, L., Rabitti, C., Maffulli, N., \& Denaro, V. (2011). Tuberculosis of the shoulder. Journal of Shoulder and Elbow Surgery, 20(4), e19-e21.

7. Narang, S. (2012). Tuberculosis of the entheses. International orthopaedics, 36(11), 2373 2378.

8. Cheng, J., Feng, S., Lei, H., Huo, W., \& Feng, H. (2019). Tuberculosis of acromioclavicular joint: a case report. BMC infectious diseases, 19(1), 1-5. 